\title{
Simulation Model for Scenario Optimization of the Ready-Mix Concrete Delivery Problem
}

\author{
Mario Galić, Ivan Kraus \\ Josip Juraj Strossmayer University of Osijek \\ Faculty of Civil Engineering Osijek \\ e-mail: mgalic@gfos.hr, ikraus@gfos.hr
}

\begin{abstract}
This paper introduces a discrete simulation model for solving routing and network material flow problems in construction projects. Before the description of the model a detailed literature review is provided. The model is verified using a case study of solving the ready-mix concrete network flow and routing problem in metropolitan area in Croatia. Within this study real-time input parameters were taken into account. Simulation model is structured in Enterprise Dynamics simulation software and Microsoft Excel linked with Google Maps. The model is dynamic, easily managed and adjustable, but also provides good estimation for minimization of costs and realization time in solving discrete routing and material network flow problems.
\end{abstract}

Key words: concrete mixture, delivery, material flow, model, network, routing, simulation, web-mapping

\section{Introduction}

Precise and right-timed delivery in construction projects' logistics is one of the significant requirements for projects' overall success. Raw materials, prefabricated elements and/or waste materials are objects of transportation to and from construction sites as daily engineering tasks. Planning such operations starts in projects' conceptual phase and finishes in realization phase with its optimization and final decision for execution. In order to integrate planning process through the projects' life cycle researchers worldwide are developing simulation and optimization algorithms and models. Aforementioned algorithms and models are mostly based on the mathematical (theoretical) models of problems developed in period of pre and after the Second World War (e.g. transportation problem (TP), vehicle routing problem (VPR), etc.) in the domain of operation research (OR). Depending on the type of the problem, which in construction industry can vary from trivial to NP-hard, there are proven sets of exact, heuristic and meta-heuristic methods applicable for solving those problems. However, the trends of developing models in scientific circles are leaning towards simulation 
models for solving logistics problems in construction practice. Previous researchers with the critical feedback of their models suggest that future models should acknowledge project's dynamic environment and its stochastic nature [1-6]. Thus, algorithms and models have to fulfil the following demands:

- precisely defined, adjustable to changes and easily controlled input parameters,

- input parameters connectable to external databases,

- transparent and user-friendly simulation model's interface and environment,

- evolutionary simulation and optimization approach,

- scenario recording and export of results.

Taking into account aforementioned demands authors developed a discrete simulation model for solving routing and network material flow problems in construction projects. The model is verified through case study experiment of solving the concrete mixture network flow and routing problem in metropolitan area taking into account real-time input parameters from construction company in Osijek, Croatia.

\section{Literature review of the routing and network flow models}

Since Hitchcock in his work [7] in 1941 introduced a new mathematical model which he named "Transportation Problem" (TP) and defined it as a linear program for solving transportation of some good from multiple sources to multiple destinations with objective function minimization of transportation costs, researchers worldwide through its application for solving similar problems in various industries are modifying the original TP model and developing new models. As defined, TP is not concerned by any other related aspect of the transportation process except transportation costs with ensured balanced model (i.e. total supply must satisfy the total demand). Thus, authors Dantzig and Ramser proposed the Vehicle Routing Problem (VRP) in their paper [8] which is considered today as "... one of the most studied combinatorial optimization problems and is concerned with the optimal design of routes to be used by a fleet of vehicles to serve a set of customers" [9, p.1]. VRP represents the problem how to optimally route a fleet of identical vehicles, at starting point centrally based, to supply destinations with known demands subject to vehicle capacity constraints. This problem was trigger for mathematician Kwan Mei-Ko [10] in 1962 to combine VRP and arc routing problem (ARP) originally recognized by Euler, and structure a new model called "Chinese Postman Problem" (CPP). CPP can be described as problem of finding a tour which passes through every edge at least once and does this in the shortest possible way. CPP was a breakthrough for many researchers in that scientific area: in 1974 Orloff [11] suggested a "Rural Postman Problem" (RPP) which is considered to be an equivalent to "Traveling Salesman Problem" (TSP); authors Frederickson et al. [12] developed a Min-Max k-Chinese Postman Problem (MM k-CPP) with the main goal to find $k$ tours, starting and ending in the depot node, such that every edge is covered by at least one tour and the length of the longest tour is minimized; while Golden and Wong [13] in 1981 suggest a model called "Capacitated Arc Routing problem" (CARP) which has the objective to find a number of tours such that each arc with positive demand is served by exactly one vehicle, the sum of demands of those arcs served by each vehicle does not exceed capacity and the total cost of the tours is minimized. 
The mentioned problems are widely recognized in construction practice. In this paper authors are focusing on problems and solutions suggested by previous authors of the ready-mix concrete (RMC) network flow and routing.

Authors Asbach et al. [14] structured a model and solved the logistical NP-hard problem of delivering concrete from concrete-producing depots to concrete-demanding customers. Their conclusion was that it is not possible to gain a deterministic solution by using the mixed integer programming solver, rather to be solved by heuristic approach and that further optimization models should anticipate the changes of input parameters to ensure more realistic and quality output data. In 2010 Schmid et al. [15] suggested a hybrid metaheuristic model for the delivery of ready-mixed concrete to construction sites. Conclusion of the aforementioned research is that by combination of the exact solution methods and Variable Neighborhood Search approach (VNS) is applicable for solving medium sized real-time instances of concrete delivery problems. Authors Maghrebi et al. [16, 17] suggested robust heuristic method and sequential heuristic method for solving large-scale RMC problems using the genetic algorithms (GA) for successful solving large scale ready-mix concrete delivery problems. Same authors in their article [18] tested the dispatchers' accuracy for solving addressed problems comparing them to mathematical optimization models which showed high accuracy of experts' decisions, but confirmed the need of optimization tool as well.

Galić et al. [19, 20] developed a simulation model for the optimized "just-in-time" production, transport and hot-mix asphalt paving, which is by its nature considered similar to the aforementioned problems. As a continuation of the developing and suggested methods, algorithms and models authors have structured a simulation model for solving discrete routing and material network flow common in construction projects.

\section{Discrete simulation model for solving routing and material network flow problems}

\subsection{Routing and material network flow problem formulation}

Transportation of goods from multiple sources to multiple sinks is well known set of problems with belonging solving methods, algorithms, models and techniques. Logical extension of the mentioned problems is the routing problem.

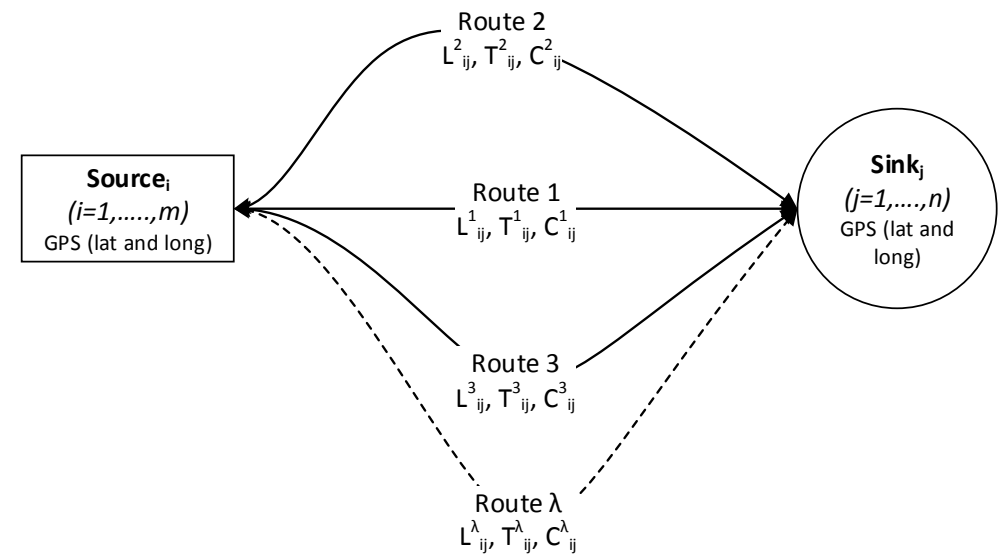

Figure 1: Graphical presentation of the routing and material network flow problems 
Except satisfying the demands of the sinks, or clearances of the sources, it is necessary to structure, define and provide optimal and suboptimal routes (scenarios) of the material network flow program. As shown in figure 1, graphical presentation of the addressed problem is consisted of defined number and belonging GPS coordinates for sources $(\mathrm{i}=1, \ldots, m)$ and sinks $(\mathrm{j}=1, \ldots, n)$, and all feasible routes $(1 \ldots . \lambda)$ with belonging parameters (i.e. distance denoted as " $L_{i j}$ ", travel time denoted as " $T_{i j}$ " and transportation costs " $C_{i j}$ "). However, addition of the GPS coordinates alone isn't significant enchantment to the simulation model. Thus, it is necessary to use those parameters as input parameters for web-mapping through online mapping servers in order to enable the dynamic nature of input parameters for the simulation and optimization.

\subsection{Simulation model}

\subsubsection{Description of the model and input parameters}

Simulation model is structured in Enterprise Dynamics simulation software (ED), which has been proven as a successful tool for simulation of similar discrete processes in construction and other industries [19-21]. One of the main advantages of ED, reported by the previous researchers, is the simple connectivity of the simulation model with the external data bases via common software for data management (i.e. Microsoft Excel, Microsoft Access). Therefore, authors structured input parameters for the simulation model in Excel file (table 1), while the interface of the simulation model is used merely to run and control the simulation; and export the output data.

Table 1: Matrix of sources' and sinks' with global coordinates and network of distances in Microsoft Excel file

\begin{tabular}{|c|c|c|c|c|c|c|}
\hline & \multicolumn{4}{|c|}{ Sinks } \\
\hline & & & Sink1 & Sink2 & Sink3 & $\operatorname{Sink}_{n}$ \\
\hline & & & $\mathrm{GPS}_{1}$ & $\mathrm{GPS}_{2}$ & $\mathrm{GPS}_{3}$ & $\mathrm{GPS}_{\mathrm{n}}$ \\
\hline \multirow{5}{*}{ Sources } & Source1 & $\mathrm{GPS}_{1}$ & $L_{11}$ & $L_{12}$ & $L_{13}$ & $L_{l n}$ \\
\hline & Source2 & $\mathrm{GPS}_{2}$ & $L_{21}$ & $L_{22}$ & $L_{23}$ & $L_{2 n}$ \\
\hline & Source3 & $\mathrm{GPS}_{3}$ & $L_{31}$ & $L_{32}$ & $L_{33}$ & $L_{3 n}$ \\
\hline & Source4 & $\mathrm{GPS}_{4}$ & $L_{41}$ & $L_{42}$ & $L_{43}$ & $L_{4 n}$ \\
\hline & Source $_{\mathrm{m}}$ & $\overline{\mathrm{GPS}_{\mathrm{m}}}$ & $L_{m 1}$ & $L_{m 2}$ & $L_{m 3}$ & $L_{m n}$ \\
\hline
\end{tabular}

Distances between sources and sinks $\left(L_{i j}\right)$ are gained by the Excel function:

$$
=\text { getGoogleDistance }\left(G P S_{i} ; G P S_{j}\right)
$$

It is necessary to underline that distances provided by the function (i.e. getGoogleDistance $\left.\left(G P S_{i} ; G P S_{j}\right)\right)$ are expressed in miles and attached to the Google maps window which can easily be embedded in the document (by allowing the external source connection in Excel's Visual Basic). Other parameters are estimated travel times $\left(T_{i j}\right)$ which is calculated by the estimation of the average velocity on that route $\left(v_{\lambda}\right)$ :

$$
T_{i j}=L_{i j} / v_{\lambda}
$$


Estimated travel time is important input parameter for pre-simulation risk assessment depending on the type of the material which is subject of the transportation (e.g. cooling time of the hot and warm mix asphalt mixtures, mixing time of the concrete, etc.). Usually constraints of transportation times are suggested or even regulated by legislation $\left(T_{\text {constrained }}\right)$.

$$
T_{i j} \leq T_{\text {constrained }}
$$

Transportation costs $\left(C_{i j}\right)$ are subject of calculation or bidding process, and can consist of material cost (common in situations when material manufacturer is also providing the transportation or material costs significantly varies among sources). It is expressed in units of material per unit of transport length:

$$
C_{i j}=C_{\text {transportation }}+C_{\text {material }}
$$

The aim of the simulations is to gain the scope of feasible scenarios from which user can differentiate optimal program $(Z)$ from the sub-optimal scenarios. Of course, optimality is determined with two objective functions: minimal total costs $\left(\mathrm{f}_{1}(\mathrm{x})\right)$ and minimal total execution time of the program $\left(\mathrm{f}_{2}(\mathrm{x})\right)$. As well, priorities of the objective functions have to be determined due to the bi-criterial final decision.

$$
Z \rightarrow \min \left(\mathrm{f}_{1}(\mathrm{x}) ; \mathrm{f}_{2}(\mathrm{x})\right)
$$

\subsubsection{Interface of the simulation model in ED}

Simulation model is consisted of two identically named files (in order to enable their connectivity): simulation model (i.e. Model.mod) and input parameters (i.e. Model.xls). Simulation model is structured of six system entities, or as it is defined in ED "atoms"; atom

\begin{tabular}{|c|c|c|}
\hline Source $\rightarrow$ & Batch & Queue \\
\hline Out: 0 & Util: $0 \%$ & 0 \\
\hline
\end{tabular}
for connection with input parameters, three results export atoms and data recording atom (figure 2).

\begin{tabular}{l} 
Transportati \\
\hline Util: $0 \%$ \\
\hline
\end{tabular}

\begin{tabular}{|l|l|}
\hline Concrete pour & Site \\
\hline Util: $0 \%$ & 7 \\
\hline
\end{tabular}

Excel Activex

Figure 2: Interface of the simulation model in ED [22] 
Each atom is system's sub-processes and it is defined by belonging input parameters:

- Source - it is usual entry into the system, which is in this model defined as the production, or storage of the material. In case of storage (depot) it is necessary to define the total quantity. While in case of production it is defined by the inter-arrival time (timed needed to finalize defined units of material), production time for the first unit, queue discipline in case of the multiple channels and if wanted trigger on exit (function by which is possible to call or initialize other atoms in the model).

- Batch + Queue2 - these two atoms are used for loading the transportation vehicle in batches and queuing them in case of more than one transportation vehicle and for loading.

- Transportation - this server is the model of the transportation process and it is defined by external input parameters (i.e. setup time, cycle time, destination atom, input strategy, and triggers for the system from MS Excel file).

- Excel AtiveX - is bypass atom for connection between simulation model file and input parameters Excel file. After the connection is established, input parameters are called up by simple input code in the belonging atom - ExcelActiveX_Read $(a, b)$ - " $a$ " is the row number, " $b$ " is the column number of the belonging cell in the Excel file.

- Concrete pouring - atom for simulation of concrete pouring on construction site defined by the time distribution.

- Sink - usual exit atom which is connected with the atoms for results export (atom "Table").

- Data Recording - atom used to collect results from passing product atoms. The user can define which results for which products should be collected and whether to write to a table and/or to Excel.

As it is common to processes simulation software, simulation time has to be defined before of the simulation initialization. In case of the robust models authors suggest to speed up the simulation to maximum due to the relatively long total simulation period.

\section{Scenario optimization of the ready-mix concrete delivery - case study}

\subsection{Description of the case study problem}

In this paper authors choose a common problem of production and delivery of ready-mix concrete from various concrete plants to multiple construction sites, in order to experimentally verify the simulation model. The network is consisted of five construction sites and four potential concrete plants, in metropolitan area in city of Osijek, Croatia. The task is to provide web-mapping simulation model of routing and concrete flow network of various scenarios.

\subsection{Input parameters for scenario simulation}

Input parameters of the case study problem have been collected from a construction company in Osijek. Five construction sites with the belonging demands for the same type of concrete mixture at the same time are the parameters provided by the construction company. 
Aforementioned construction company owns one of the potential concrete plants and has its own truck mixers. Other three potential concrete plants are outsourced in order to fulfil the total need for concrete mixtures of all sites at the same time. Construction sites, as well as the plants, are located in Osijek's metropolitan area (figure 3) and they all fulfil the constraint of maximal distance of $50 \mathrm{~km}$ between plants and sites.

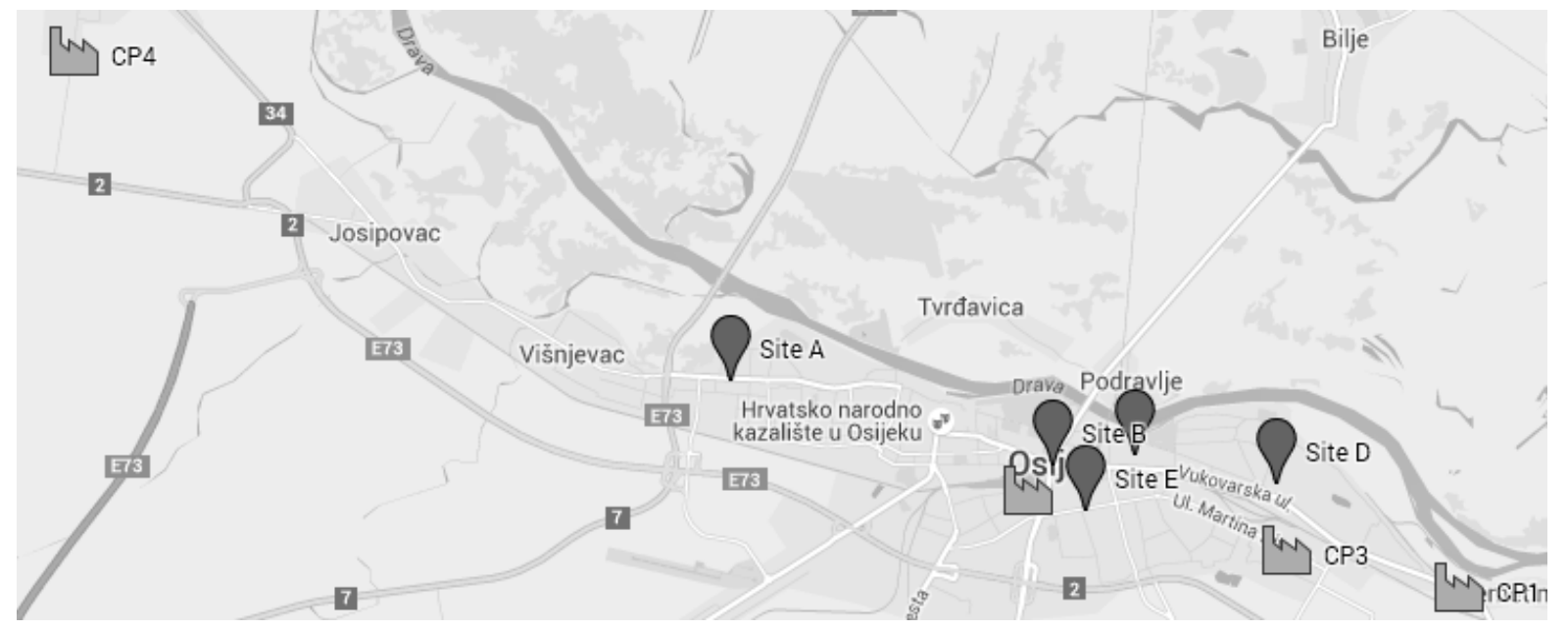

Figure 3: Locations of the case study plants and construction sites in Google maps [23]

Transportation costs were given by the aforementioned construction company while the costs of concrete per unit were estimated as equal at all concrete plants and therefore in the model they were taken as constant, meaning that they were excluded from the total costs calculation. The web map is embedded into the Excel document where all the input parameters have to be attached to belonging cells in order to bypass the real-time parameters to simulation model (table 2).

Table 2: Matrix form of the case study input parameters for the $1^{\text {st }}$ simulation in Microsoft Excel file

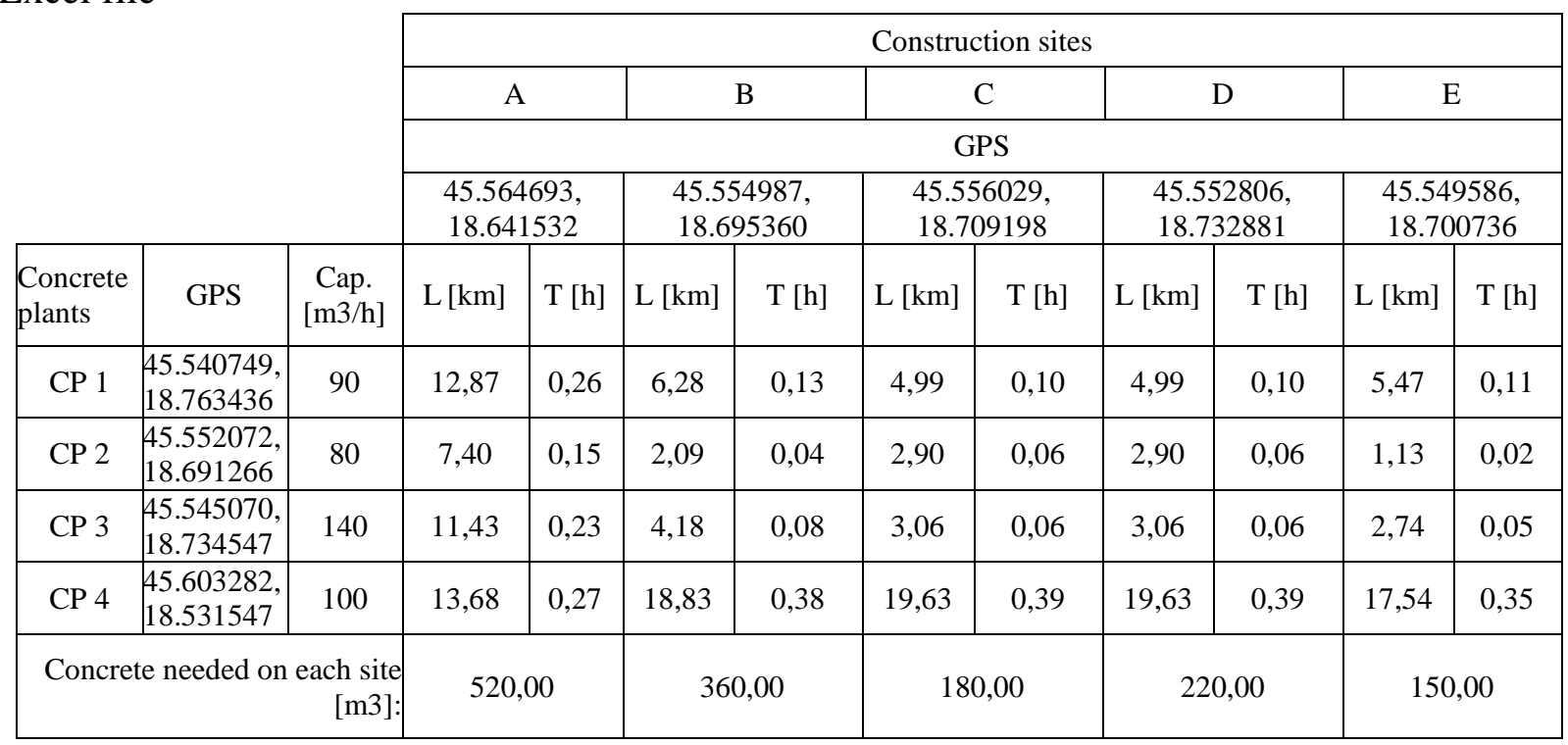




\subsection{Scenario simulation}

\subsubsection{First route simulation}

First simulation used the shortest route for the entire network from the Google maps. Results are exported and stored in the same Excel file for input parameters, but in different sheet. Except earlier elaborated input parameters, authors have estimated batch size (i.e. truck mixer capacity) of $6 \mathrm{~m}^{3}$ and uniform distribution of concrete pouring on construction site $15-20 \mathrm{~m}^{3} / \mathrm{h}$ which was used for all three simulations sets. Simulation model ready for $1^{\text {st }}$ route scenario is shown in figure 4.

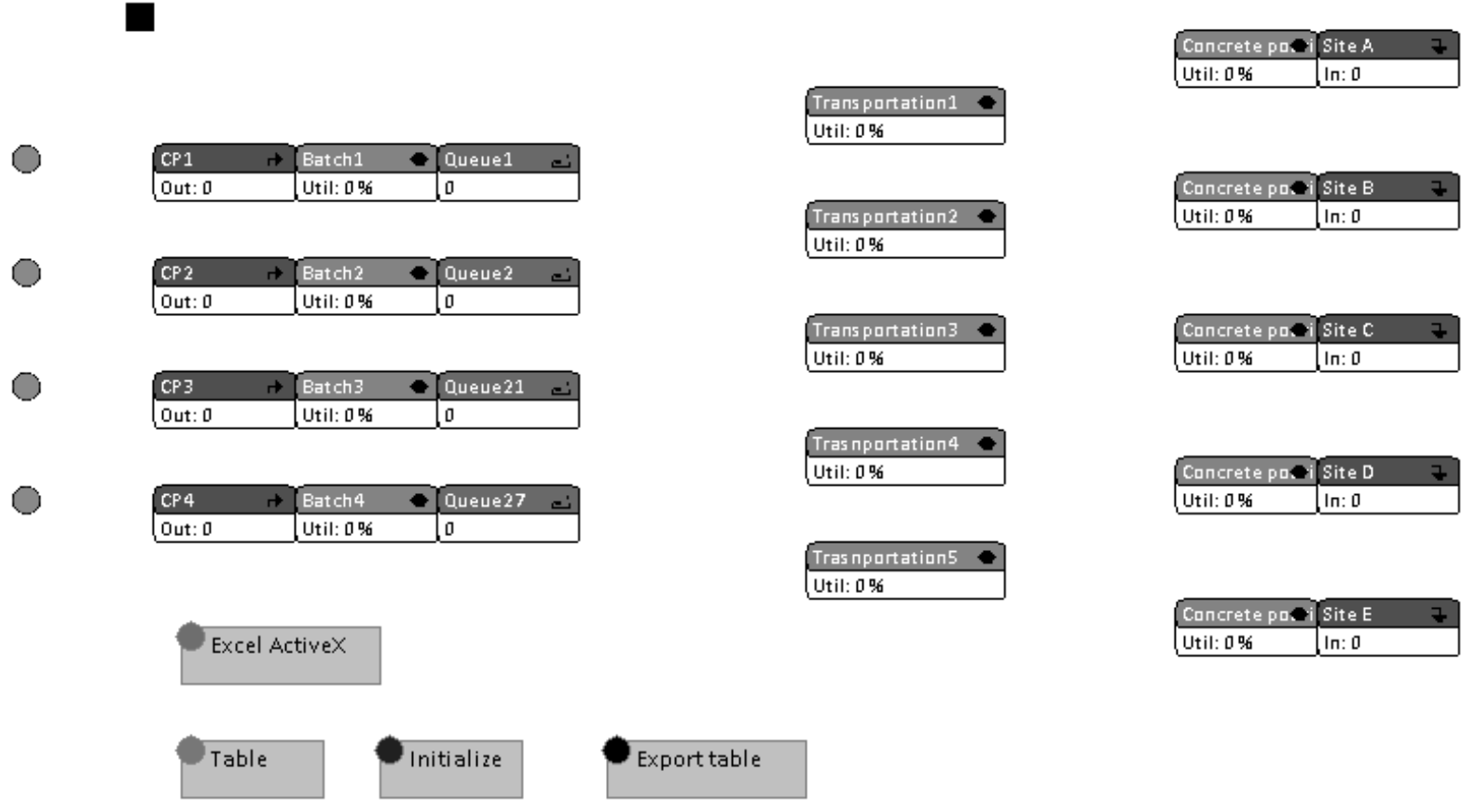

Figure 4: Simulation model ready for $1^{\text {st }}$ route scenario simulation [22]

Simulation time was very short $(9 \mathrm{~s})$ due to the fact of relatively small size problem. Total execution time for completing the task on all construction sites in this simulation set is $30 \mathrm{~h}$ $38 \mathrm{~min}$. Table 3 presents results in matrix form of the concrete distribution production and transportation of the first simulation. Total transportation costs of the concrete delivery program are $8.176 €$.

Table 3: Results of the $1^{\text {st }}$ simulation - program of the concrete production and delivery in $\mathrm{m}^{3}$

\begin{tabular}{|l|c|c|c|c|c|}
\hline & Site A & Site B & Site C & Site D & Site E \\
\hline CP1 & 112 & 84 & 54 & 60 & 30 \\
\hline CP2 & 180 & 48 & 30 & 78 & 24 \\
\hline CP3 & 102 & 114 & 54 & 36 & 60 \\
\hline CP4 & 126 & 114 & 42 & 46 & 36 \\
\hline
\end{tabular}




\subsubsection{Second route simulation}

In the second simulation set, authors changed the routes for the entire network using the second shortest alternative route, if possible, for concrete delivery by choosing it in the embedded Google maps window in the input parameters Excel file, while the other input parameters and interface of the model remained the same.

Table 4: Results of the $2^{\text {nd }}$ simulation - program of the concrete production and delivery in $\mathrm{m}^{3}$

\begin{tabular}{|c|c|c|c|c|c|}
\hline & Site A & Site B & Site C & Site D & Site E \\
\hline CP1 & 142 & 90 & 24 & 42 & 60 \\
\hline CP2 & 138 & 84 & 72 & 60 & 6 \\
\hline CP3 & 138 & 84 & 54 & 42 & 42 \\
\hline CP4 & 102 & 102 & 30 & 76 & 42 \\
\hline
\end{tabular}

In table 4 it is obvious that concrete delivery program has change with the route change. Simulation time was the same as in the first simulation, while total execution time increased and it is $32 \mathrm{~h} 29 \mathrm{~min}$. Total transportation costs of the concrete delivery program are $8.412 €$.

\subsubsection{Third route simulation}

As in previous simulation set, in third authors have changed routes to alternative to previous. Concrete delivery program is shown in table 5. Total execution time in this scenario is $30 \mathrm{~h} 50$ min, while the total transportation costs are $8.607 €$.

Table 5: Results of the $3^{\text {rd }}$ simulation - program of the concrete production and delivery in $\mathrm{m}^{3}$

\begin{tabular}{|c|c|c|c|c|c|}
\hline & Site A & Site B & Site C & Site D & Site E \\
\hline CP1 & 114 & 120 & 24 & 36 & 60 \\
\hline CP2 & 144 & 78 & 24 & 102 & 6 \\
\hline CP3 & 136 & 84 & 84 & 36 & 36 \\
\hline CP4 & 126 & 78 & 48 & 46 & 48 \\
\hline
\end{tabular}

\subsection{Interpretation of the simulation results}

In this case authors generated three scenarios of concrete delivery. The final number of scenarios is limited by the feasible routes and their combinations. Combination of feasible routes is as well interesting for post-optimal analysis especially in projects realization phase in situations of unexpected occurrences. The result of the simulations is three scenarios, or more precisely said three programs of ready-mix concrete delivery from concrete plants to construction sites. Results represent a table of feasible scenarios with belonging two parameters for the final decision (table 6). 
Table 6: Matrix form of the simulation results

\begin{tabular}{|c|c|c|}
\hline Scenario & Total transportation costs & Total realization time \\
\hline Scenario 1 & $8.176 €$ & $30 \mathrm{~h} 38 \mathrm{~min}$ \\
\hline Scenario 2 & $8.412 €$ & $32 \mathrm{~h} 29 \mathrm{~min}$ \\
\hline Scenario 3 & $8.607 €$ & $30 \mathrm{~h} 50 \mathrm{~min}$ \\
\hline
\end{tabular}

Optimal solution, in mathematical sense, is the one which provides the minimal costs and minimal realization time (equation 5). In this case the first scenario provides such result and therefore it is logical to proclaim it as the optimal program and continue with the post-optimal analysis. However, due to the stochastic environment of the project it is necessary to consider the sub-optimal programs (scenarios).

\section{Discussion and conclusions}

Mathematical models of original problems are the platforms for solving various problems in construction industry. Concept of process modelling and simulation combines those models and therefore provides quality results, as well as the record of the optimal and suboptimal solutions if necessary. Static nature of input parameters, noted by the previous researchers and the practitioners, by this approach is reduced or even unremarked. Suggested model is experimentally proven as successful and useful tool for solving discrete routing and material network flow problems. It fulfilled the assumptions and suggestions from previous researchers: it is dynamic, easily managed, adjustable and developing model for solving the addressed and similar problems. The model is applicable for various types of loads for which transportation has similar constraints (i.e. transportation time is a hard constraint, storage of the material is not an option, the demand for the material is at the same time and in short time period has to be delivered). Generating scenarios is simple and very quick to perform through project's life cycle. One can easily generate scenarios as many as feasible, by changing the input parameters. As a result, this simulation model provides a scope of feasible scenarios of the program from which user can decide which program will be chosen for the realization. The main disadvantage of the model is sensitivity of Google maps connection and thus it is necessary to ensure continuous monitoring of the input parameters. Web maps provide a dynamic feedback about the routes and therefore strict and consistent users' monitoring is crucial. The provider of the web maps used in this model offers possibility for users to download and use offline maps. This can be useful in areas where the Internet is slow, mobile data expensive or where users cannot connect to the Internet. However, offline mode does not offer current traffic conditions what can be crucial for using this model. Authors suggest that offline mode of web maps could be applied for baseline planning and preliminary analysis. In further work authors will focus on model's commercial usage as an addition tool or extension for software available on the market. As well, the model will be expanded and modified for solving cases of delivery of different mixtures of ready-mix concrete. Furthermore, model will be verified in cases of integer and binary routing and material network flow problems. 


\section{References}

[1] AbouRizk, S. (2010). Role of simulation in construction engineering and management. Journal of Construction Engineering and Management. 136 (10), 1140-53. http://dx.doi.org/10.1061/(ASCE)CO.1943-7862.0000220.

[2] Mariz, R. N., Picchi, F. A., Granja, A. D. \& de Melo, R. S. S. (2013). Production Cells in Construction: Considering Time, Space and Information Linkages to Seek Broader Implementations. Journal of Engineering, Project, and Production Management. 3 (1), 46-55.

[3] Tang, Y., Liu, R. \& Sun, Q. (2014). Schedule control model for linear projects based on linear scheduling method and constraint programming. Automation in Construction. 37 22-37. http://dx.doi.org/10.1016/j.autcon.2013.09.008.

[4] Márquez, A. C. (2010). Dynamic modelling for supply chain management: dealing with frontend, back-end and integration issues. London: Springer Science \& Business Media.

[5] Galić, M., Dolaček-Alduk, Z. \& Završki, I. (2013). The importance of additional criteria in solving transportation problem. In Creative Construction Conference 2013, (219 - 29). Budapest: Diamond Congress, Ltd.

[6] Galić, M. \& Kraus, I. (2016). Web-mapping discrete simulation model for solving routing and material network flow problems in construction projects. In 8th International Scientific Conference of Civil Engineering and Architecture for PhD Students and Young Scientists below 35 years old, Košice, Slovakia: Technical University of Košice, Faculty of Civil Engineering.

[7] Hitchcock, F. L. (1941). The distribution of a product from several sources to numerous localities. J Math phys. 20 (2), 224-30. http://dx.doi.org/10.1002/sapm1941201224.

[8] Dantzig, G. B. \& Ramser, J. H. (1959). The truck dispatching problem. Management Science. 6 (1), 80-91. http://dx.doi.org/10.1287/mnsc.6.1.80.

[9] Baldacci, R., Battarra, M. \& Vigo, D. (2008). Routing a heterogeneous fleet of vehicles. In Golden, B., Raghavan, S. \& Wasil, E. (Eds.), The vehicle routing problem: latest advances and new challenges (3-27). Springer.

[10] Kwan, M.-K. (1962). Graphic programming using odd or even points. Chinese Math. 1 (110), 273-7.

[11] Orloff, C. (1974). A fundamental problem in vehicle routing. Networks. 4 (1), 35-64. http://dx.doi.org/10.1002/net.3230040105.

[12] Frederickson, G. N., Hecht, M. S. \& Kim, C. E. (1976). Approximation algorithms for some routing problems. In 17th Annual Symposium on Foundations of Computer Science, (216-27). Houston: IEEE.

[13] Golden, B. L. \& Wong, R. T. (1981). Capacitated arc routing problems. Networks. 11 (3), 30515. http://dx.doi.org./10.1002/net.3230110308.

[14] Asbach, L., Dorndorf, U. \& Pesch, E. (2009). Analysis, modeling and solution of the concrete delivery problem. European Journal of Operational Research. 193 (3), 820-35. http://dx.doi.org/10.1016/j.ejor.2007.11.011.

[15] Schmid, V., Doerner, K. F., Hartl, R. F. \& Salazar-González, J.-J. (2010). Hybridization of very large neighborhood search for ready-mixed concrete delivery problems. Computers \& Operations Research. 37 (3), 559-74. http://dx.doi.org/10.1016/j.cor.2008.07.010. 
[16] Maghrebi, M., Waller, T. S. \& Sammut, C. Scheduling concrete delivery problems by a robust meta heuristic method. In Modelling Symposium (EMS), 2013 European, (375-80). IEEE.

[17] Maghrebi, M., Travis Waller, S. \& Sammut, C. (2014). Sequential Meta-Heuristic Approach for Solving Large-Scale Ready-Mixed Concrete-Dispatching Problems. Journal of Computing in Civil Engineering. 30 (1), http://dx.doi.org/10.1061/(ASCE)CP.1943-5487.0000453.

[18] Maghrebi, M., Travis Waller, S. \& Sammut, C. (2014). Assessing the accuracy of expert-based decisions in dispatching ready mixed concrete. Journal of Construction Engineering and Management. 140 (6), 06014004. http://dx.doi.org/10.1061/(ASCE)CO.1943-7862.0000853.

[19] Galić, M., Završki, I. \& Dolaček-Alduk, Z. (2016). Scenario simulation model for optimized allocation of construction machinery. Građevinar. 68 (02), 105-12. http://dx.doi.org/10.14256/JCE.1462.2015.

[20] Galić, M., Thronicke, R., Schreck, B. M., Feine, I. \& Bargstädt, H.-J. (2015). Process modeling and scenario simulation in construction using Enterprise Dynamics simulation software. e-GFOS. 10 (1), 22-9. http://dx.doi.org/10.13167/2015.10.3.

[21] Weber, J. (2008). Simulation von Logistikprozessen auf Baustellen auf Basis von 3D-CAD Daten. Dotrmund, Germany.

[22] INCONTROL Simulation Solutions (2014). Enterprise Dynamics [computer software]. The Netherlands: INCONTROL Simulation Solutions.

[23] Google Inc. Google Maps. https://www.google.hr/maps/@ 45.5720848,18.604127,12z/data=!3m1!4b1!4m2!6m1!1 szI3BNYVBM EqA.kmtD-GzDJCMs?hl=hr. (2.2.2016.) 